\title{
Detection of Methicillin-Resistant Coagulase-Negative Staphylococci and Surveillance of Antibacterial Resistance in a Multi-Center Study from Iran
}

\author{
Mohammad Reza Nahaei ${ }^{1,2}$; Mohammad Reza Shahmohammadi ${ }^{3,4,5}$; Shiva Ebrahimi ${ }^{6}$; \\ Morteza Milani ${ }^{1,7,}$ \\ ${ }^{1}$ Drug Applied Research Center, Tabriz University of Medical Sciences, Tabriz, IR Iran \\ 2 Department of Microbiology, School of Medicine, Tabriz University of Medical Sciences, Tabriz, IR Iran \\ ${ }^{3}$ Shabestar Hospital, Tabriz University of Medical Sciences, Tabriz, IR Iran \\ 4 alinasab Hospit, Tabriz University of Medical Sciences, Tabriz, IR Iran \\ 5 Aalinasab Hospital, Tabriz University of Medical Sciences, Tabriz, IR Iran \\ Department of Microbiology, Ahar Branch, Islamic Azad University, Ahar, IR Iran
6 Imam Reza Hospital, Faculty of Medicine, Tabriz University of Medical Sciences, Tabriz, IR Iran \\ ${ }^{7}$ School of Advanced Medicine, Tabriz University of Medical Sciences, Tabriz, IR Iran \\ *Corresponding author: Morteza Milani, School of Advanced Medicine, Tabriz University of Medical Sciences, Tabriz, IR Iran. Tel/Fax:+98-4113364661, E-mail: mohammadmilano@gmail.com
}

Received: May 3, 2014; Revised: June 16, 2014; Accepted: July 24, 2014

\begin{abstract}
Background: Coagulase-negative staphylococci (CNS) are a common cause of nosocomial infections. In recent years, an increase in the incidence of methicillin-resistant coagulase-negative staphylococci (MRCNS) has led to the severity of the disease.

Objectives: The aim of this study was to isolate and identify MRCNS strains by oxacillin disk agar diffusion, oxacillin agar screening, and polymerase chain reaction (PCR) and to evaluate their antibacterial resistance patterns.

Patients and Methods: Totally, 122 CNS isolates were collected from the clinical specimens of four hospitals in Iran. Susceptibility testing was performed by disk agar diffusion against 15 antimicrobial agents. Then, disk agar diffusion, agar screening, and PCR were applied to determine susceptibility to oxacillin.

Results: Out of the 122 isolates, 92 isolates were found to be MRCNS by PCR. The sensitivities and specificities of disk agar diffusion and agar screening were $89.2 \%$ and $69 \%$ and $93.8 \%$ and $96.3 \%$, respectively. Also, 93 CNS isolates were resistant to Methicillin according to disk agar diffusion.

Discussion: Our results indicated that agar screening was superior to oxacillin disk agar diffusion. A comparison between the antibiotic sensitivity patterns of the MRCNS and the Methicillin-Susceptible Coagulase-Negative Staphylococci (MSCNS) showed that the MRCNS were predominantly multiple-drug resistant isolates as the simultaneous resistance rate to 4 or more antibiotics in the MRCNS and MSCNS was $93 \%$ and $56 \%$, respectively.
\end{abstract}

Keywords: Coagulase; Staphyloccus; Methicillin Resistance; Methicillin Resistance

\section{Background}

Coagulase-negative staphylococci (CNS) are a common cause of nosocomial infections. Because these organisms are a normal commensal on the skin and the differentiation of the infection from colonization and contamination may be difficult, the epidemiological studies of CNS infection is complicated (1). The types of CNS infections in children are broad and well described. Pediatric oncology patients, bone marrow transplant recipients, and children with burns are at high risk for acquiring CNS infections (2). The most frequently encountered CNS species associated with human infections is Staphylococcus epidermidis, especially in association with intravascular catheters. Other CNS species such as S. saprophyticus are involved in a variety of infections. This bacterium is an important pathogen in human urinary tract infections, especially in young females (3).

The development of antibiotic-resistant bacteria has increased at a frightening rate since the introduction of antibiotics in the 1940s. The frequent overuse of antibiotics, incorrect diagnoses, inappropriate prescribing, noncompliance with antibiotic therapy by patients, and the misuse of antibiotics have all promoted the rapid spread of resistance even to modern antibacterial agents (4). Methicillin-resistance in staphylococci is mediated by the mecA gene, which encodes for the penicillin-binding protein $2 \mathrm{~A}$ (PBP2A), resulting in decreased affinity for the $\beta$-lactam antibiotics (5). This gene is widely disseminated among Staphylococcus species, which might be due to horizontal transmissions among CNS isolates and S. aureus $(6,7)$. Serious nosocomial infections caused by methicillin-resistant staphylococci (MRS) require the administration of non- $\beta$-lactam antibiotics. An increase in MRS in recent years has led to the severity of the disease caused. The high cost of antibiotics and increasing resistance to

Copyright (C) 2015, Ahvaz Jundishapur University of Medical Sciences. This is an open-access article distributed under the terms of the Creative Commons Attribution-NonCommercial 4.0 International License (http://creativecommons.org/licenses/by-nc/4.0/) which permits copy and redistribute the material just in noncommercial usages, provided the original work is properly cited. 
Nahaei MR et al.

them justify the identification, prevention, and control of these infections.

\section{Objectives}

The purpose of this study was to evaluate methicillinresistant coagulase-negative staphylococci (MRCNS) isolates with three methods, consisting of oxacillin disk agar diffusion, oxacillin agar screening, and polymerase chain reaction (PCR). Also, the surveillance of the antibacterial resistance patterns of our isolates was our next objective.

\section{Patients and Methods}

\subsection{Sample Collection}

In the present study,122 CNS isolates were obtained from various clinical specimens, including the blood, urine, wound, and body fluids. These samples were collected at four different centers in Iran, including Pediatric hospital of Tehran (No.1), Imam Reza hospital (No. 2), Aalinasab hospital of Tabriz (No. 3), and Fatemiyyeh hospital of Shabestar (No. 4) from July 2012 to December 2013.

\subsection{Isolation and Identification of Coagulase-Neg- ative Staphylococci}

In this study, all of the isolates were identified as CNS by different biochemical tests such as Gram staining, catalase, coagulase tube test, ability to growth on mannitol salt agar, deoxyribonuclease (DNase), and novobiocin resistance test (8). Staphylococcus aureus ATCC 29213 (oxacillin susceptible) and S. aureus ATCC 33591 (oxacillin resistant) were used as negative and positive controls, respectively.

\subsection{Antimicrobial Susceptibility Testing}

The antimicrobial susceptibility of CNS and the reference strains (S. aureus ATCC 29213 and ATCC 33591) were determined by the conventional disk agar diffusion test according to The Clinical and Laboratory Standards Institute (CLSI) recommendations (9). Briefly, disks of different antibiotics (Mast, UK), including oxacillin $(1 \mu \mathrm{g} /$ disk), penicillin ( $5 \mu \mathrm{g} /$ disk), erythromycin $(15 \mu \mathrm{g} /$ disk$)$, clindamycin $(2 \mu \mathrm{g} /$ disk $)$, cefazolin $(30 \mu \mathrm{g} /$ disk $)$, rifampicin $(5 \mu \mathrm{g} /$ disk $)$, ceftriaxone $(30 \mu \mathrm{g} /$ disk $)$, sulfamethoxazole/trimethoprim (1.25 - 23.75 $\mu \mathrm{g} /$ disk), tetracycline (30 $\mu \mathrm{g} /$ disk $)$, fusidic acid (10 $\mu \mathrm{g} /$ disk), ciprofloxacin $(30 \mu \mathrm{g} /$ disk), vancomycin (30 $\mu \mathrm{g} / \mathrm{disk})$, teicoplanin (30 $\mu \mathrm{g} /$ disk), amikacin (30 $\mu \mathrm{g} /$ disk), and gentamicin (10 $\mu \mathrm{g} /$ disk), were placed on the plates and incubated for 24 hours and examined for inhibition zones.

\subsection{Oxacillin Agar Screening}

All the isolates were cultured on Mueller-Hinton agar (Pronadisa, Spain) supplemented with $4 \%$ (wt/vol) $\mathrm{NaCl}$ comprising oxacillin (Sigma, Australian) at concentra- tions of $0.6,4$, or $6 \mu \mathrm{g} / \mathrm{mL}$. The agar plates were inoculated by the swabbing of the surface with adjusted inoculums suspensions (0.5 McFarland) (9). Oxacillin resistance was confirmed by bacterial growth after 24 and 48 hours of incubation at $35^{\circ} \mathrm{C}$.

\subsection{DNA Extraction}

The genomic DNA of the total isolates was extracted via the Cetyltrimethylammonium Bromide (CTAB) method (10) and stored at $20^{\circ} \mathrm{C}$. A loopful of bacteria was added to sterile distilled water $(1.5 \mathrm{ml})$, mixed gently, and was centrifuged in $1000 \mathrm{~g}$ for 10 minutes. The supernatant was discarded and Tris-EDTA buffer (TE buffer) $(270 \mu \mathrm{L})$ plus sodium dodecyl sulfate (SDS) $10 \%(30 \mu \mathrm{L}$ ) plus proteinase $\mathrm{K}(5 \mu \mathrm{L})$ was added to a microtube and then incubated for 1 hour at $65^{\circ} \mathrm{C}$. Afterward, $\mathrm{M} \mathrm{NaCl}$ solution (100 $\mu \mathrm{L}$ ) was added to the microtube and mixed well. Then, prewarmed $\mathrm{CTAB} / \mathrm{NaCl}\left(65^{\circ} \mathrm{C}\right)$ solution $(80 \mu \mathrm{L})$ was added to the microtube and incubated at $65^{\circ} \mathrm{C}$ for 10 minutes. Chloroform-isoamylalcohol $(24: 1)$ solution $(700 \mu \mathrm{L})$ was added to the microtube and vortexed for 20 seconds. The suspension was centrifuged in $12,000 \mathrm{~g}$ for $5-10$ minutes at $10^{\circ} \mathrm{C}$ and the aqueous phase was transferred into a new microtube. Thereafter, isopropanol $(200-300 \mu \mathrm{L})$ was added to each microtube and mixed gently and incubated at $20^{\circ} \mathrm{C}$ for 30 minutes before it was finally centrifuged in 12,000 $\mathrm{g}$ for 10 minutes. The supernatant was discarded, and the pellet was resuspended in $70 \%$ cold ethanol $(1 \mathrm{ml})$ and then centrifuged in 12,000 $\mathrm{g}$ for 5 minutes at $10^{\circ} \mathrm{C}$. The supernatant was discarded and after air drying, the DNA pellet was dissolved in TE $(10: 1)$ buffer $(50 \mu \mathrm{L})$ and incubated at $37^{\circ} \mathrm{C}$ for 30 minutes before it was stored at $4^{\circ} \mathrm{C}$ overnight.

\subsection{Detection of the mecA Gene by Polymerase Chain Reaction Method}

A partial mecA gene was amplified using mecA gene primers (mecA-F: 5'-AAAATCGATGGTAAAGGTTGGC-3' and mecA-R: 5'-AGTTCTGCAGTACCGGATTTGC-3'), which were selected on the basis of the published nucleotide sequence $(11,12)$. The PCR reaction mixture was prepared in a reaction mixture $(50 \mu \mathrm{L})$, containing Tris- $\mathrm{HCl}(10$ $\mathrm{mmol} / \mathrm{L}), 0.2 \mathrm{mmol} / \mathrm{L}$ of each deoxynucleotide, $\mathrm{MgCl}_{2}(1.5$ $\mathrm{mmol} / \mathrm{L}), 25 \mathrm{pmol}$ of each primer, and Taq polymerase (2.5 units) (CinnaGen, Iran). PCR amplification consisted of one cycle at $94^{\circ} \mathrm{C}$ for 3 minutes, followed by 30 cycles at $94^{\circ} \mathrm{C}$ for 30 seconds, $55^{\circ} \mathrm{C}$ for 30 seconds, $72^{\circ} \mathrm{C}$ for $30 \mathrm{sec}-$ onds, and then finally $72^{\circ} \mathrm{C}$ for 3 minutes. For the analysis of the mecA gene, primers mecA-F and mecA-R yielded a fragment of $533 \mathrm{bp}$. Therefore, the PCR products were examined using 2\% agarose gel (Kawsar Biotech Company, Iran) electrophoresis. The gels were stained with ethidium bromide (Geneon Company, Germany) to detect of the fragment of the mecA gene. Also, the 100 bp plus DNA ladder (Fermentas Company, Iran) was used as a DNA molecular weight standard. 


\section{Results}

In this study, 122 CNS strains (110 S. epidermidis and $12 \mathrm{~S}$. saprophyticus isolates) were isolated from various samples, including 66 (54.1\%), 41 (33.6\%), 12 (9.8\%), and $3(2.5 \%)$ isolates from blood, urine, body fluid, and wound specimens, respectively. Also among the 122 CNS, 66 strains were separated from females and 56 strains from males. Ninety-three (76.3\%) CNS isolates were resistant to oxacillin (MRCNS) according to disk agar diffusion. Also, oxacillin agar screening using three concentrations was performed for all the isolates, the results of which are shown in Table1. In our study, the presence of the mecA gene was investigated in 92 (74.5\%) of the isolates by PCR (Figure 1).

Our results demonstrated that simultaneous resistance to several antibiotics in the mecA-negative isolates was less than that of the mecA-positive isolates. In other words, the mecA-negative isolates had a high level of susceptibility to all the antibiotics other than penicil- lin. The results of our study also showed that all the CNS were susceptible to vancomycin and no strains resistant to this antibiotic were observed by disk agar diffusion. For example, according to the comparison of antibiotic resistance patterns between the mecA-positive and mecA-negative isolates, as is depicted in Table 2, 96.3\% of the mecA-negative isolates and $100 \%$ of the mecA-positive isolates were resistant to penicillin. In this study, 10 (8.2\%) outpatient isolates and $82(67.2 \%)$ patient isolates revealed mecA gene, which was statistically significant $(\mathrm{P}<0.05)$. Information regarding the source of the isolates and their resistance patterns is presented in Table 3. Although $90 \%$ of the isolates were recovered from Aalinasab hospital, no significant association was detected between the strains isolated from the different hospitals and this gene.

Table 1. Results of the Oxacillin Susceptibility Patterns and mecA Gene in the Coagulase-Negative Staphylococci Isolates

\begin{tabular}{|c|c|c|c|c|c|c|}
\hline \multirow{3}{*}{ Method } & \multicolumn{4}{|c|}{ Number of Isolates } & \multirow{3}{*}{ Sensitivity ${ }^{\mathrm{a}}$} & \multirow{3}{*}{ Specificity $^{\mathrm{a}}$} \\
\hline & \multicolumn{2}{|c|}{ mecA Positive $(\mathrm{n}=92)$} & \multicolumn{2}{|c|}{ mecA Negative $(n=30)$} & & \\
\hline & True Positive & False Positive & True Negative & False Negative & & \\
\hline Disk agar diffusion & 83 & 9 & 20 & 10 & 89.2 & 69 \\
\hline Agar screen, $0.6 \mu \mathrm{g} / \mathrm{mL}$ & 92 & 0 & 24 & 6 & 93.9 & 100 \\
\hline Agar screen, $4 \mu \mathrm{g} / \mathrm{mL}$ & 92 & 0 & 24 & 6 & 94 & 100 \\
\hline Agar screen, $6 \mu \mathrm{g} / \mathrm{mL}$ & 89 & 3 & 24 & 6 & 93.7 & 88.9 \\
\hline
\end{tabular}

a Data are presented as \%.

Figure 1. Amplified Products of the mecA Gene by Polymerase Chain Reaction

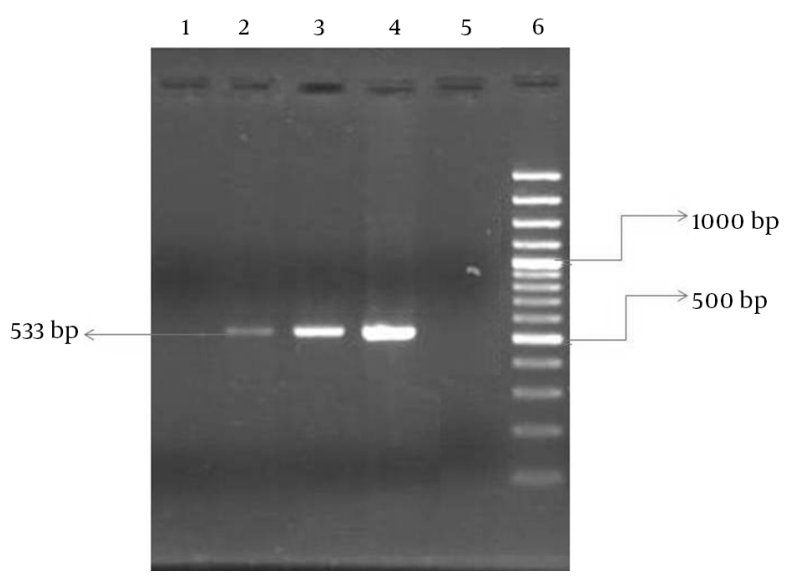

Lane 1: clinical isolate of CNS mecA negative, lanes 2 and 3: clinical isolates of CNS mecA positive, lane 4: positive control, lane 5: negative control, lane 6: 100 - 3,000 bp DNA ladder.
Table 2. Frequency of the Antibiotic Resistance of the mecAPositive and mecA-Negative Isolates

\begin{tabular}{|c|c|c|}
\hline $\begin{array}{l}\text { Antimicrobial } \\
\text { Agents }\end{array}$ & $\begin{array}{c}\text { mecA-Positive } \\
(\mathbf{n}=\mathbf{9 2})^{\mathrm{a}}\end{array}$ & $\begin{array}{c}\text { mecA-Negative } \\
(\mathbf{n}=\mathbf{3 0})^{\mathrm{a}}\end{array}$ \\
\hline Oxacillin & $83(90.2)$ & $10(33.4)$ \\
\hline Penicillin & $92(100)$ & $22(73.3)$ \\
\hline Rifampicin & $17(18.5)$ & $2(6.7)$ \\
\hline Erythromycin & $78(84.8)$ & $15(50)$ \\
\hline Clindamycin & $53(57.6)$ & $7(23.2)$ \\
\hline Cefazolin & $32(34.7)$ & $1(3.3)$ \\
\hline Ceftriaxone & $75(81.5)$ & $10(33.3)$ \\
\hline Co-trimoxazole & $67(72.8)$ & $13(43.4)$ \\
\hline Gentamicin & $50(54.4)$ & $2(6.6)$ \\
\hline Ciprofloxacin & $44(48.9)$ & $5(16.6)$ \\
\hline Vancomycin & $0(0)$ & $0(0)$ \\
\hline Fusidic acid & $23(25)$ & $6(20)$ \\
\hline Tetracycline & $56(60.9)$ & $13(43.4)$ \\
\hline Amikacin & $24(26.1)$ & $2(6.6)$ \\
\hline Teicoplanin & $7(7.6)$ & $0(0)$ \\
\hline
\end{tabular}

${ }^{\mathrm{a}}$ Values are presented as No(\%). 
Nahaei MR et al.

\begin{tabular}{|c|c|c|c|c|}
\hline \multirow{2}{*}{ Antimicrobial Agents } & \multicolumn{4}{|c|}{ Hospitals } \\
\hline & No. $1(n=61)$ & $\operatorname{No.} 2(n=19)$ & $\operatorname{No.3}(n=20)$ & No. $4(n=22)$ \\
\hline Oxacillin & $44(72.1)$ & $14(73.7)$ & $19(95)$ & $16(72.7)$ \\
\hline Penicillin & $59(96.7)$ & $16(84.2)$ & $20(100)$ & $19(86.4)$ \\
\hline Rifampicin & $8(13.1)$ & $3(15.8)$ & $5(25)$ & $3(13.6)$ \\
\hline Erythromycin & $49(80.3)$ & $12(63.1)$ & $19(95)$ & $14(63.6)$ \\
\hline Clindamycin & $32(52.4)$ & $7(36.8)$ & $14(70)$ & $7(31.8)$ \\
\hline Cefazolin & $17(27.8)$ & $3(15.8)$ & $8(40)$ & $6(27.3)$ \\
\hline Ceftriaxone & $47(77)$ & $11(57.9)$ & $14(70)$ & $13(59.1)$ \\
\hline Co-trimoxazole & $38(52.3)$ & $14(73.7)$ & $16(80)$ & $12(54.5)$ \\
\hline Gentamicin & $28(45.9)$ & $10(52.6)$ & $10(50)$ & $4(18.2)$ \\
\hline Ciprofloxacin & $20(32.8)$ & $9(47.4)$ & $14(70)$ & $7(31.8)$ \\
\hline Vancomycin & $0(0)$ & $0(0)$ & $0(0)$ & $0(0)$ \\
\hline Fusidic acid & $10(16.4)$ & $6(31.6)$ & $4(20)$ & $9(40.1)$ \\
\hline Tetracycline & $30(49.2)$ & $11(57.9)$ & $16(80)$ & $12(54.5)$ \\
\hline Amikacin & $15(24.6)$ & $4(21)$ & $5(25)$ & $2(9.1)$ \\
\hline Teicoplanin & $6(9.8)$ & $0(0)$ & $1(5)$ & $0(0)$ \\
\hline
\end{tabular}

${ }^{a}$ Values are presented as No (\%).

\section{Discussion}

Numerous studies have been conducted to determine the optimal methods for the phenotypic detection of methicillin resistance in CNS $(13,14)$. In this study, we compared two phenotyping methods and genotyping methods to evaluate the oxacillin susceptibilities of CNS. We found that disk agar diffusion showed the lowest sensitivity and specificity (89.2\% and $69 \%$, respectively) for the detection of oxacillin resistance between the two methods (Table 1) inasmuch as 9 isolates that contained the mecA gene were found to be susceptible to oxacillin and 10 isolates that did not contain the mecA gene were found to be resistant to oxacillin by disk diffusion method. This finding may be associated with heteroresistance to oxacillin and the absence and presence of the mecA gene expression in these isolates $(15,16)$. The detection of oxacillin resistance among CNS isolates is difficult mainly because it is often heterogeneous. Over the years, various methods have been employed to overcome this particular obstacle (15).

The use of agar screening at a concentration of $6 \mu \mathrm{g} / \mathrm{mL}$ of oxacillin and $24-48$ hours of incubation is no longer recommended by the CLSI. However, many studies have observed that this technique is sensitive and can be used as an additional test to acknowledge the results obtained by disk agar diffusion and PCR $(14,17)$. In our study, the results obtained by this method showed a good relation with those obtained by PCR (93.7\% sensitivity and $88.9 \%$ specificity). Our results showed that agar screening at a concentration of $4 \mu \mathrm{g} / \mathrm{ml}$ and $0.6 \mu \mathrm{g} / \mathrm{mL}$ of oxacillin and 24 - 48 hours of incubation presented $94 \%$ sensitivity and
$100 \%$ specificity. Not only is this test the most accurate method for the detection of oxacillin resistance among CNS isolates but also it is easy to perform and cheap, which makes it a suitable alternative to PCR.

Agar screening at concentrations of 6,4 , and $0.6 \mu \mathrm{g} /$ $\mathrm{mL}$ of oxacillin and $24-48$ hours of incubation, as is described here, conferred a reliable detection of the mecA-positive and mecA-negative CNS clinical isolates. The accurate diagnosis of MRCNS strains in hospitals, patients, and health care workers is an important need, and continuous surveillance of antibiotic resistance patterns among CNS strains should receive due attention from health care systems (18). Antibiotic-resistant CNS has emerged as a major cause of morbidity and mortality in the hospital setting during the last decade. Many studies from different parts of the world have reported the presence of multidrug resistance in CNS (19). A comparison between the antibiotic sensitivity patterns of the MRCNS and the methicillin-susceptible coagulase-negative staphylococci (MSCNS) in our study revealed that the MRCNS had a higher level of resistance to many antibiotics than the MSCNS. Many authors have reported similar findings showing higher antibiotic resistance among MRCNS isolates $(12,18,20)$.

The results of our study indicated that the three most effective drugs against the MRCNS were vancomycin, teicoplanin, and rifampicin. Vancomycin is the last resort and drug of choice to treat infections caused by MRCNS isolates in the world; therefore, the emergence of resistance to vancomycin could be a serious concern for pub- 
Nahaei MR et al.

lic health $(21,22)$. Fortunately, vancomycin exhibited $100 \%$ effectiveness, which chimes in with the results of previous studies $(22,23)$. During the last decade, S. aureus and CNS have emerged as important nosocomial pathogens, and the rising antibiotic resistance in these organisms is a major public health concern $(23,24)$. The recognition and discrimination of $S$. aureus and CNS and the detection of methicillin resistance are essential for prompting effective antimicrobial therapy and for limiting the unnecessary use of certain antibiotics (25). Our results revealed that the MRCNS and MSCNS isolates were most resistant to penicillin, with a resistance frequency of $100 \%$ and $73.3 \%$, respectively. This finding is in agreement with other reports from Iran and other countries (26-28). We observed that the resistance rate to different antibiotics among the MRCNS strains was higher than those sensitive to methicillin, which is consistent with the results of another study (29). In addition, multidrug resistance rates in our MRCNS and MSCNS isolates were $93 \%$ and $56 \%$, respectively. Other published reports have indicated a closely similar or lower percentage of resistance (30-32).

The majority of the strains isolated from the Pediatric hospital of Tehran and Aalinasab hospital of Tabriz were MRCNS, while the number of the MRCNS and MSCNS isolated from the other hospitals was almost equal. These findings were statistically significant $(\mathrm{P}<0.05)$. MRCNS are emerging nosocomial pathogens and every effort should be made to control and prevent infections. Effective infection control programs should be devised and the risk factors should be minimized through regular surveys of health care providers to detect and treat the CNS carriers (18). The current study showed that $76.2 \%$ of the CNS isolated in Iran hospitals during a 6-month period were resistant to methicillin. The results also revealed an increase in the number of MRCNS with reduced susceptibility to fusidic acid, rifampicin, and teicoplanin.

\section{Acknowledgements}

We would like to thank the staff of the hospitals involved and the microbiology laboratory of Tabriz university of medical sciences for their assistance in conducting this study.

\section{Authors' Contributions}

Development of the original idea: Mohammad R. Nahaei and Mohammad R. Shahmohammadi. Study concept and design: Mohammad R. Nahaei, Mohammad R. Shahmohammadi and Morteza Milani. Analysis and interpretation of the data: Morteza Milani. Preparation of the manuscript: Mohammad R. Shahmohammadi, Morteza Milani and Shiva Ebrahimi. Revision of the manuscript: Mohammad R. Nahaei.

\section{Funding/Support}

This study was supported as an Msc thesis by Ahar Branch, Islamic Azad university, which had no role in the design and performance of the study; collection, management, and analysis of the data; or preparation, review, and approval of the manuscript.

\section{References}

1. Nnis System . National Nosocomial Infections Surveillance (NNIS) System Report, data summary from January 1992 through June 2003, issued August 2003. Am J Infect Control. 2003;31(8):481-98.

2. Santucci SG, Gobara S, Santos CR, Fontana C, Levin AS. Infections in a burn intensive care unit: experience of seven years. J Hosp Infect. 2003;53(1):6-13.

3. Heikens E, Fleer A, Paauw A, Florijn A, Fluit AC. Comparison of genotypic and phenotypic methods for species-level identification of clinical isolates of coagulase-negative staphylococci. J Clin Microbiol. 2005;43(5):2286-90.

4. Otto M. Virulence factors of the coagulase-negative staphylococci. Front Biosci. 2004;9:841-63.

5. Rohrer S, Bischoff M, Rossi J, Berger-Bachi B. Mechanisms of methicillin resistance.Wymondham: MRSA Current Perspectives, Caister Academic Press; 2003.

6. Archer GL, Niemeyer DM. Origin and evolution of DNA associated with resistance to methicillin in staphylococci. Trends Microbiol. 1994;2(10):343-7.

7. Pierre J, Williamson R, Bornet M, Gutmann L. Presence of an additional penicillin-binding protein in methicillin-resistant Staphylococcus epidermidis, Staphylococcus haemolyticus, Staphylococcus hominis, and Staphylococcus simulans with a low affinity for methicillin, cephalothin, and cefamandole. Antimicrob Agents Chemother. 1990;34(9):1691-4.

8. Klein E, Smith DL, Laxminarayan R. Hospitalizations and deaths caused by methicillin-resistant Staphylococcus aureus, United States, 1999-2005. Emerg Infect Dis. 2007;13(12):1840-6.

9. Wayne P. Performance Standards for Antimicrobial Susceptibility Testing; Ninth Informational Supplement. NCCLS Document M100-S9. 2008

10. Sambrook J, Russell DW. Molecular cloning: a laboratory manual. Woodbury. 2001

11. Vaez H, Tabaraei A, Moradi A, Ghaemi EA. Evaluation of methicillin resistance Staphylococcus aureus isolated from patients in Golestan province-north of Iran. Afr J Microbiol Res. 2011;5(4):432-6.

12. Rahimi F, Bouzari M, Maleki Z, Rahimi F. Antibiotic susceptibility pattern among Staphylococcus spp. with emphasis on detection of mecA gene in methicillin resistant Staphylococcus aureus isolates. Arch Clin Infect Dis. 2009;4(3):143-50.

13. Rabelo MA, Bezerra Neto AM, Silva ECBF, Oliveira WLM, Melo FL, Lopes ACS, et al. Phenotypic methods for determination of methicillin resistance in Staphylococcus spp. from health care workers. Jornal Brasileiro de Patologia e Medicina Laboratorial. 2013;49(2):91-6.

14. Najar Peerayeh S, Azimian A, Mostafaee M, Siadat SD. Identification of methicillin-resistant Staphylococcus aureus by disk diffusion method, determination of MIC and PCR for mecA gene. Modares J Med Sci Pathobiol. 2009;12(3):61-9.

15. Chambers HF. Methicillin resistance in staphylococci: molecular and biochemical basis and clinical implications. Clin Microbiol Rev. 1997;10(4):781-91.

16. Frebourg NB, Nouet D, Lemee L, Martin E, Lemeland JF. Comparison of ATB staph, rapid ATB staph, Vitek, and E-test methods for detection of oxacillin heteroresistance in staphylococci possessing mecA.J Clin Microbiol. 1998;36(1):52-7.

17. Cavassini M, Wenger A, Jaton K, Blanc DS, Bille J. Evaluation of MRSA-Screen, a simple anti-PBP 2a slide latex agglutination kit, for rapid detection of methicillin resistance in Staphylococcus aureus. J Clin Microbiol. 1999;37(5):1591-4.

18. Nikbakht M, Nahaei MR, Akhi MT, Asgharzadeh M, Nikvash S. Nasal carriage rate of staphylococcus aureus in hospital personnel and inpatients and antibiotic resistance pattern of isolated strains from nasal and clinical specimens in tabriz. Med J Tabriz Uni Med Sci.2007;29(2) 
19. Brzychczy-Wloch M, Borszewska-Kornacka M, Gulczynska E, Wojkowska-Mach J, Sulik M, Grzebyk M, et al. Prevalence of antibiotic resistance in multi-drug resistant coagulase-negative staphylococci isolated from invasive infection in very low birth weight neonates in two Polish NICUs. Ann Clin Microbiol Antimicrob. 2013;12:41.

20. Sharma V, Jindal N, Devi P. Prevalence of methicillin resistant coagulase negative staphylococci in a tertiary care hospital. Iran J Microbiol. 2010;2(4):185-8.

21. Vahdani P, Saifi M, Aslani MM, Asarian AA, Sharafi K. Antibiotic resistant patterns in MRSA isolates from patients admitted in ICU and infectious ward. Tanaffos. 2004;3(11):37-44.

22. Rahimi F, Bouzari M, Katouli M, Pourshafie MR. Antibiotic Resistance Pattern of Methicillin Resistant and Methicillin Sensitive Staphylococcus aureus Isolates in Tehran, Iran. Jundishapur J Microbiol. 2013;6(2)

23. Saderi H, Owlia P, Nadoushan MRJ. PP-007 Difference in epidemiology and antibiotic susceptibility of methicillin resistant and methicillin susceptible Staphylococcus aureus isolates. Int J Infect Dis. 2010;14:S26.

24. Diekema DJ, BootsMiller BJ, Vaughn TE, Woolson RF, Yankey JW, Ernst EJ, et al. Antimicrobial resistance trends and outbreak frequency in United States hospitals. Clin Infect Dis. 2004;38(1):78-85.

25. Zhang K, Sparling J, Chow BL, Elsayed S, Hussain Z, Church DL, et al. New quadriplex PCR assay for detection of methicillin and mupirocin resistance and simultaneous discrimination of Staphylococcus aureus from coagulase-negative staphylococci.
Clin Microbiol. 2004;42(11):4947-55.

26. Davoodi NR, Yousefi JV, Harzandi N, Hajrafi A, Rajaei B, GerayeshNejad S, et al. Molecular detection of methicillin resistant Staphylococcus aureus (MRSA) and methicillin resistant coagulase-negative Staphylococcus (CoNS) in Iran. Afr J Microbiol Res. 2012;6(16):3716-21.

27. Shanthi M, Sekar U. Antimicrobial susceptibility pattern of methicillin resistant staphylococcus aureus at sri ramachandra medical centre. Sri Ramachandra J Med. 2009;2(2)

28. Moosavian M, Wadowsky R. Analysis of genomic fingerprint patterns of coagulase-negative Staphylococci strains isolated from pediatric patients blood cultures using repetitive sequencebased PCR. Jundishapur J Microbiol. 2010;2010(4, Autumn):147-53.

29. Tahnkiwale SS, Roy S, Jalgaonkar SV. Methicillin resistance among isolates of Staphylococcus aureus: antibiotic sensitivity pattern \& phage typing. Indian J Med Sci. 2002;56(7):330-4.

30. Assadullah S, Kakru DK, Thoker MA, Bhat FA, Hussain N, Shah A. Emergence of low level vancomycin resistance in MRSA. Indian J Med Microbiol. 2003;21(3):196-8.

31. Ruhe JJ, Smith N, Bradsher RW, Menon A. Community-onset methicillin-resistant Staphylococcus aureus skin and soft-tissue infections: impact of antimicrobial therapy on outcome. Clin Infect Dis. 2007;44(6):777-84.

32. Japoni A, Alborzi A, Orafa F, Rasouli M, Farshad S. Distribution patterns of methicillin resistance genes (mecA) in Staphylococcus aureus isolated from clinical specimens. Iran Biomed $\mathrm{J}$. 2004;8(4):173-8. 